\title{
FUNCTIONAL-INTEGRAL SOLUTION FOR THE SCHRÖDINGER EQUATION WITH POLYNOMIAL POTENTIAL: A WHITE NOISE APPROACH
}

\author{
S. MAZZUCCHI
}

\begin{abstract}
A functional integral representation for the weak solution of the Schrödinger equation with polynomially growing potentials is proposed in terms of a white noise functional.
\end{abstract}

Key words: Feynman path integrals, Schrödinger equation, white noise analysis, polynomial potential.

AMS classification : 35C15, 35Q40, 28C20, 60H40, 35Q40, 81Q05,47D06, 35B60.

\section{INTRODUCTION}

The study of the functional integral representation for the solution of the Schrödinger equation

$$
\left\{\begin{array}{l}
i \hbar \frac{\partial}{\partial t} \psi=-\frac{\hbar^{2}}{2 m} \Delta \psi+V \psi \\
\psi(0, x)=\psi_{0}(x), \quad x \in \mathbb{R}^{d}
\end{array}\right.
$$

in particular in connection with the mathematical definition of Feynman path integrals [12], is a largely studied topic ${ }^{1}[20,3,19,26]$.

The realization of the heuristic Feynman path integral representation for the time evolution of the wave function

$$
\psi(t, x)=\int_{\gamma(t)=x} e^{\frac{i}{\hbar} \int_{0}^{t}\left(\dot{\gamma}(\tau)^{2} / 2 m-V(\gamma(\tau))\right) d \tau} \psi(0, \gamma(0)) d \gamma
$$

in terms of a well defined functional integral, presents particular difficulties because of the oscillatory nature of the integration. Indeed the

\footnotetext{
${ }^{1}$ see also the special issue [ J. Math. Phys. 36 (1995), no. 5 ] of J. Math. Phys. on functional integration.
} 
presence of the imaginary unit $i$ in the exponent appearing in the integrand does not allow the realization of "Feynman's measure", heuristically written as:

$$
d \mu_{F}(\gamma)=\frac{e^{\frac{i}{\hbar} \int_{0}^{t}\left(\dot{\gamma}(\tau)^{2} / 2 m\right) d \tau} d \gamma}{\int e^{\frac{i}{\hbar} \int_{0}^{t}\left(\dot{\gamma}(\tau)^{2} / 2 m\right)} d \tau} d \gamma
$$

in terms of a complex bounded variation measure on a suitable space of paths $\gamma:[0, t] \rightarrow \mathbb{R}^{d}[7]$.

Consequently mathematicians tried to realize the "integral with respect to the Feynman's measure", i.e. the heuristic expression

$$
\int f(\gamma) d \mu_{F}(\gamma)
$$

just in terms of a linear continuous functional ("Feynman functional") on a suitable linear space (or even better, a Banach algebra) of "integrable functions" $f$.

Several approaches to the rigorous mathematical realization of the Feynman functional have been proposed, for instance by means of analytic continuation of Gaussian Wiener integrals [7, 10, 21, 27, 2, 20], or as limit of finite dimensional approximations by implementing a suitable version of the Trotter product formula $[27,13,14]$, or via infinite dimensional oscillatory integrals $[11,1,26]$, or as an infinite dimensional distribution, in the framework of white noise calculus $[19,24,8$, $16,17,18]$.

The main problem, which is common to all the existing approaches, is the limitation on the class of "integrable functions" and, consequently, on the class of potentials $V$ for which it is possible a rigorous mathematical realization of formula (2). In fact all the existing approaches can handle very well potentials $V$ which are the sum of an harmonic oscillator part, plus a bounded perturbation which is the Fourier transform of a complex bounded measure on $\mathbb{R}^{d}$, but the study of other unbounded physically interesting potentials presents some difficulties.

The case of exponentially growing potentials which are Laplace transforms of measures has been handled in the framework of analytically continued Wiener integrals [2] and by means of white noise analysis $[22]$. In this case (as well as in the previously mentioned one) the perturbative Dyson series is convergent.

The case of unbounded potentials with polynomial growth at infinity, when the degree of the polynomial is strictly greater than 2, presents further difficulties. First of all the perturbative Dyson series is not 
convergent, but it is only asymptotic, even if some results of Borel summability have been obtained [6] (see also [31] for Borel summability of corresponding asymptotic series for the eigenvalues). Moreover it has been proved [32] that for time-independent superquadratic polynomial potentials the fundamental solution of the time dependent Schrödinger equation is nowhere of class $C^{1}$, as a function of the time $t$ and the space variables. In other words there is not even the hope to provide, in terms of a functional integral, a well defined function of the time and the space variables, which can be differentiated in order to prove that it is the fundamental solution of the Schrödinger equation with polynomial potentials.

Nevertheless some partial results have been obtained in $[4,5,25]$ concerning the representation of the solution of the Schrödinger equation with a quartic oscillator potential in terms of infinite dimensional oscillatory integrals. An analytically-continued Wiener integral representation for the weak solution of the Schrödinger equation (in the sense of equation (13)) has been proposed. The same representation has been generalized in [6] to more general polynomial potentials.

In [10] an analytically continued Feynman-Kac formula has been proposed. It provides a functional integral representation for the pointwise solution of the Schrödinger equation also in the case of polynomial potentials of the form

$$
V(x)=\sum_{n=1}^{4 M+2} a_{n} x^{n}, \quad x \in \mathbb{R}^{d},
$$

with $a_{n}, n=1, \ldots 4 M+2$ multilinear symmetric applications from $\left(\mathbb{C}^{d}\right)^{n}$ to $\mathbb{C}$ such that $a_{n}\left(\left(\mathbb{R}^{d}\right)^{n}\right) \subset \mathbb{R}$ and $a_{4 M+2} \in \mathbb{R}, a_{4 M+2} x^{4 M+2}<0$ for all $x \in \mathbb{R}^{d}$ if $M \geq 1$, while $a_{2} x^{2}<\frac{|x|^{2}}{4 t^{2}}$ if $M=0$, (where $|x|=$ $\sup _{j=1, \ldots d}\left|x_{j}\right|$, for $\left.x \in \mathbb{C}^{d}\right)$.

Analogous results, with the further inclusion of a linear time-dependent potential, have been obtained in [17], where the Feynman integrand, formally $e^{\frac{i+1}{2} \int_{0}^{t} \dot{\gamma}(\tau)^{2} d \tau-i \int_{0}^{t} V(\gamma(\tau) d \tau}$, has been defined as an infinite dimensional Hida distribution, in the framework of white noise analysis. A pointwise-defined (unbounded) evolution system on a space of holomorphic functions on an open subset of $\mathbb{C}^{d}$, corresponding to the time evolution described by the Schrödinger equation, is defined in terms of an analytically-continued Wiener integral and interpreted in terms of a white noise distributional pairing.

The present paper extends the results of [17] to homogeneous polynomial potentials of the form

$$
V(x)=a_{4 M} x^{4 M}, \quad x \in \mathbb{R}^{d}, \quad M \in \mathbb{N},
$$


with $a_{4 M}$ is a completely symmetric multilinear application from $\left(\mathbb{C}^{d}\right)^{4 M}$ to $\mathbb{C}$ such that $a_{4 M}\left(\left(\mathbb{R}^{d}\right)^{4 M}\right) \subset \mathbb{R}$.

For a suitable class of holomorphic functions $\phi, \psi \in L^{2}\left(\mathbb{R}^{d}\right)$ and potentials $V$ (possibly time-dependent), a sesquilinear form $I_{t}^{\xi}(\phi, \psi)$ is constructed by means of an analytically-continued Wiener integral, and interpreted as the weak solution of the Schrödinger equation (in the sense of equation (13)). We prove that $I_{t}^{\xi}(\phi, \psi)$ is given by the Ttransform of a white noise distribution, which can be interpreted as the "Feynman integrand". In the case of polynomial potentials of the form (3), by applying the methods of [6], it is shown that $I_{t}^{\xi}(\phi, \psi)$ is equal to the matrix element of an unitary propagator $U(t, 0)$ on $L^{2}\left(\mathbb{R}^{d}\right)$.

The paper is organized as follows. Section 2 gives an overview of the mathematical realization of the Feynman integrand in terms of white noise distributions. Section 3 presents the construction of the functional $I_{t}^{\xi}(\phi, \psi)$ and proves that it can be interpreted as the T-transform of an Hida distribution. Section 4 studies the special case of polynomial potentials of the form (3). In the whole paper, for notational simplicity, we shall put $\hbar=m=1$.

\section{The Feynman integrand AS A White nOISE Distribution}

The white noise approach to the mathematical definition of Feynman path integrals realizes the heuristic integral $\int e^{\frac{i}{2} \int_{0}^{t} \dot{\gamma}(\tau)^{2} d \tau} f(\gamma) d \gamma$ as a distributional pairing on a well defined infinite dimensional measure space of paths (see, e.g., [19, 24, 8, 16, 17, 18]). The idea can be simply explained in a finite dimensional setting. Indeed by considering formally the following integrals on $\mathbb{R}^{n}$, it is possible to write a chain of equalities

$$
\begin{aligned}
& (2 \pi i)^{-n / 2} \int_{\mathbb{R}^{n}} e^{\frac{i}{2}(x, x)} f(x) d x \\
= & (2 \pi i)^{-n / 2} \int_{\mathbb{R}^{n}} e^{\frac{i}{2}(x, x)+\frac{1}{2}(x, x)} f(x) e^{-\frac{1}{2}(x, x)} d x \\
= & \frac{1}{i^{n / 2}} \int_{\mathbb{R}^{n}} e^{\frac{i}{2}(x, x)+\frac{1}{2}(x, x)} f(x) d \mu_{G}(x),
\end{aligned}
$$

where the latter line can be interpreted as the distributional pairing of $i^{-n / 2} e^{\frac{i}{2}(x, x)+\frac{1}{2}(x, x)}$ and $f$, not with respect to Lebesgue measure $d x$ on $\mathbb{R}^{n}$, but rather with respect to the standard Gaussian measure $\mu_{G}$ on $\mathbb{R}^{n}$.

This idea can be generalized to the infinite dimensional case and applied to the definition of the Feynman integrand by replacing $\left(\mathbb{R}^{n}, \mu_{G}\right)$ with the white noise space $\left(S_{d}^{\prime}, \mathcal{B}, \mu\right)$ constructed in the following way. 
Let $L_{d}^{2}$ be the real Hilbert space

$$
L_{d}^{2}:=L^{2}(\mathbb{R}) \otimes \mathbb{R}^{d}
$$

which is isomorphic to the direct sum $d$ copies of the real Hilbert space $L^{2}(\mathbb{R}, d x), d x$ denoting the Lebesgue measure on $\mathbb{R}$. Let us denote the norm on $L_{d}^{2}$ by $|\xi|_{0}=\left(\sum_{i=1}^{d} \int_{\mathbb{R}}\left|\xi_{i}(x)\right|^{2} d x\right)^{1 / 2}$, where $\xi_{i}, i=1, \ldots, d$, is the $i$ th component of the vector $\xi \in L_{d}^{2}$.

Let us consider correspondingly the nuclear vector space $S_{d}:=S(\mathbb{R}) \otimes$ $\mathbb{R}^{d}, S(\mathbb{R})$ being the space of Schwartz test functions, equipped with a family of Hilbert norms $\left\{||_{p}\right\}_{p}$ topologizing it. Let us consider its dual $S_{d}^{\prime}:=S^{\prime}(\mathbb{R}) \otimes \mathbb{R}^{d}$ (with $S^{\prime}(\mathbb{R})$ being the space of tempered Schwartz distributions) and the Gaussian measure $\mu$ on the $\sigma$-algebra $\mathcal{B}$ generated by the cylindrical sets, defined via Minlos' theorem [23] by its characteristic functional:

$$
\xi \in S_{d} \mapsto \int_{S_{d}^{\prime}} e^{i\langle\xi, \omega\rangle} d \mu(\omega)=e^{-\frac{|\xi|_{0}^{2}}{2}},
$$

where $\langle\xi, \omega\rangle$ is the distributional pairing between $\xi \in S_{d}$ and $\omega \in S_{d}^{\prime}$. $\left(S_{d}^{\prime}, \mathcal{B}, \mu\right)$ is called (d-dimensional) white noise space.

By unitary equivalence, the mapping

$$
\xi \in S_{d} \mapsto\langle\xi, \cdot\rangle
$$

can be extended to the vectors $\xi \in L_{d}^{2},\langle\xi, \cdot\rangle$ denoting a random variable on $\left(S_{d}^{\prime}, \mathcal{B}, \mu\right)$. Let us recall, as an example, that a version of the $d-$ dimensional Brownian motion is given by

$$
\left.B_{\tau}(\omega):=\left(\left\langle 1_{[0, \tau]} \otimes e_{1}, \omega\right\rangle, \ldots, 1_{[0, \tau]} \otimes e_{d}, \omega\right\rangle\right),
$$

where $\left\{e_{1}, \ldots, e_{d}\right\}$ is a basis of $\mathbb{R}^{d}, 1_{[0, \tau]}$ is the characteristic function of the interval $[0, \tau] \subset \mathbb{R}, \omega \in \Omega \equiv S^{\prime}\left(\mathbb{R}^{d}\right)$.

Let us consider the space $L^{2}(\mu):=L^{2}\left(S_{d}^{\prime}, \mathcal{B}, d \mu\right)$ and the Gelfand triple

$$
\left(S_{d}\right) \subset L^{2}(\mu) \subset\left(S_{d}\right)^{\prime}
$$

which is constructed in an analogous way to the finite dimensional case, see [19]. The elements of $\left(S_{d}\right)^{\prime}$ are called white noise distributions, while the elements in $\left(S_{d}\right)$ are the corresponding test functions.

Given a $\xi \in S_{d}$, it is possible to construct the following white noise test function:

$$
\omega \in S_{d}^{\prime} \mapsto e^{i\langle\xi, \omega\rangle} .
$$

Consequently, for any "Hida distribution" $\Phi \in\left(S_{d}\right)^{\prime}$ the following functional $T \Phi: S_{d} \rightarrow \mathbb{C}$ is well defined:

$$
\xi \in S_{d} \mapsto T \Phi(\xi):=\left\langle\left\langle e^{i\langle\xi, \cdot\rangle}, \Phi\right\rangle\right\rangle,
$$


where $\left\langle\left\langle e^{i\langle\xi, \cdot\rangle}, \Phi\right\rangle\right\rangle$ denotes the distributional pairing between $e^{i\langle\xi, \cdot\rangle} \in$ $\left(S_{d}\right)$ and $\Phi \in\left(S_{d}^{\prime}\right)$. The functional $T \Phi$ can be heuristically written as

$$
T \Phi(\xi):=\int_{S_{d}^{\prime}} e^{i\langle\xi, \omega\rangle} \Phi(\omega) d \mu(\omega) .
$$

The application from $\left(S_{d}^{\prime}\right)$ to the space of continuous functionals on $S_{d}$ given by

$$
\Phi \in\left(S_{d}^{\prime}\right) \mapsto T \Phi, \quad T \Phi(\xi)=\left\langle\left\langle e^{i\langle\xi, \cdot\rangle}, \Phi\right\rangle\right\rangle, \quad \xi \in S_{d}
$$

is called $T$ - transform, an infinite dimensional analogue of the Fourier transform. The following basic theorem, due to Potthoff and Streit [28, 19], provides a characterization of T-transforms of Hida distributions.

Theorem 1. A functional $F: S_{d} \rightarrow \mathbb{C}$ is the T-transform of a unique Hida distribution iff the it has the following properties:

(1) For all $\xi, \eta \in S_{d}$ the mapping

$$
z \in \mathbb{R} \mapsto F(\xi+z \eta) \in \mathbb{C}
$$

has an analytic continuation to $\mathbb{C}$ as an entire function.

(2) There exist some positive constants $\alpha, \beta$ and a continuous norm \|\| on $S_{d}$ such that for all $z \in \mathbb{C}, \xi \in S_{d}$,

$$
|F(z \xi)| \leq \alpha e^{\beta|z|^{2}\|\xi\|^{2}} .
$$

A functional $F: S_{d} \rightarrow \mathbb{C}$ with the properties (1) and (2) above is called $U$-functional.

In the framework of white noise analysis, the "Feynman integrand" $e^{\frac{i}{2} \int_{0}^{t} \dot{\gamma}(\tau) d \tau}$ is realized as an Hida distribution [19, 8, 22, 24]. In particular it is possible to realize the Feynman path integral representation for the fundamental solution $G(t, x ; 0, y)$ of the Schrödinger equation over $\mathbb{R}^{d}$ :

$$
G(t, x ; 0, y)=\int_{\substack{\gamma(t)=x \\ \gamma(0)=y}} e^{\frac{i}{2 \hbar} \int_{0}^{t} \dot{\gamma}^{2}(s) d s} d \gamma, \quad t>0, x, y \in \mathbb{R}^{d}
$$

The paths $\gamma$ are modeled by:

$$
\gamma(\tau)=x-\int_{\tau}^{t} \omega(\sigma) d \sigma:=x-\left\langle 1_{(\tau, t]}, \omega\right\rangle .
$$

The Feynman integrand for the free particle is realized as the distribution

$$
I_{0}(x, t ; y, 0)(\omega)=N e^{\frac{i+1}{2} \int_{0}^{t} \omega(\tau)^{2} d s} \delta(\gamma(0)-y),
$$


(where $N$ denotes a normalization constant and $\delta(\gamma(0)-y)$ fixes the initial point of the path), defined by its T-transform

$$
\begin{gathered}
T I_{0}(x, t ; y, 0)(\xi)=\frac{1}{(2 \pi i t)^{d / 2}} \exp \left(-\frac{1}{2} \int_{-\infty}^{0} \xi(\tau)^{2} d \tau-\frac{1}{2} \int_{t}^{\infty} \xi(\tau)^{2} d \tau\right. \\
\left.-\frac{i}{2} \int_{0}^{t} \xi(\tau)^{2} d \tau+\frac{i}{2 t}\left(\int_{0}^{t} \xi(\tau) d \tau+x-y\right)^{2}\right), \quad \xi \in S_{d} .
\end{gathered}
$$

The same technique allows one to include a potential $V$ and provide, for example, the Feynman path integral representation for the solution of the Schrödinger equation for the (time dependent) harmonic oscillator [16], or for potentials $V$ that are the Fourier or the Laplace transforms of bounded measures [22].

\section{A Representation For the WeAK SOlution of the SCHRÖDINGER EQUATION}

Let us denote by $D_{\theta_{1}, \theta_{2}}$ the sector of of the Riemann surface $\tilde{\mathbb{C}}$ of the logarithm defined by:

$$
D_{\theta_{1}, \theta_{2}}:=\left\{z \in \mathbb{C}, z=\rho e^{i \phi}: \rho>0, \phi \in\left(\theta_{1}, \theta_{2}\right)\right\} .
$$

Let $C_{t}$ be the Banach space of continuous paths $B:[0, t] \rightarrow \mathbb{R}^{d}$ with $B(0)=0$, endowed with the sup-norm and let $W$ be the Wiener measure of it.

Let $\mathcal{H}_{t}$ denote the Hilbert space of absolutely continuous paths $\gamma$ : $[0, t] \rightarrow \mathbb{R}^{d}$ with $\gamma(0)=0$ and with weak derivative $\dot{\gamma}$ in $L^{2}([0, t])$, endowed with the inner product $\left(\gamma_{1}, \gamma_{2}\right)=\int_{0}^{t} \dot{\gamma}_{1}(\tau) \dot{\gamma}_{2}(\tau) d \tau$. $\left(\mathcal{H}_{t},(),\right)$ is the reproducing kernel Hilbert space of the Wiener space $\left(C_{t}, W\right)$ $[15,23]$. Analogously let us consider the Hilbert space product $\mathbb{R}^{d} \times \mathcal{H}_{t}$, endowed the natural inner product

$$
\left(\left(x_{1}, \gamma_{1}\right),\left(x_{2}, \gamma_{2}\right)\right)=x_{1} \cdot x_{2}+\int_{0}^{t} \dot{\gamma}_{1}(\tau) \dot{\gamma}_{2}(\tau) d \tau
$$

and the Banach space $\mathbb{R}^{d} \times C_{t}$, endowed with the product measure $d x d W, d x$ being the Lebesgue measure on $\mathbb{R}^{d}$.

Let us consider two vectors $\phi, \psi \in L^{2}\left(\mathbb{R}^{d}\right)$ satisfying the following condition.

Assumption 1. For any $x \in \mathbb{R}^{d}$ the functions

$$
z \mapsto \bar{\phi}(z x), \quad z \mapsto \psi(z x), \quad z \in \bar{D}_{0, \frac{\pi}{4}}
$$

are continuous on $\bar{D}_{0, \frac{\pi}{4}}$ and holomorphic on $D_{0, \frac{\pi}{4}}$. 
This assumption is quite strong, but as we shall see in section 4 it is quite simple to find a dense set of vectors in $L^{2}\left(\mathbb{R}^{d}\right)$ fulfilling this condition.

Let us consider a real potential $V(\tau)$, possibly depending explicitly on the time variable $\tau$, satisfying the following conditions.

\section{Assumption 2.}

a) for any $\tau \in[0, t]$, the function $V(\tau): \mathbb{R}^{d} \rightarrow \mathbb{R}$ is continuous and admits an analytic continuation to the subset $D \subset \mathbb{C}^{d}$, given by

$$
D=\left\{z \in \mathbb{C}^{d}, z=e^{i \pi / 4} x, x \in \mathbb{R}^{d}\right\} .
$$

b) the map

$$
(t, x) \in[0, t] \times \mathbb{R}^{d} \mapsto V\left(\tau, e^{i \pi / 4} x\right)
$$

is continuous.

Remark 1. This assumption could be weakened. For instance one can consider potentials $V$ which are continuous on $R^{d} \backslash N$, where $N$ is a null capacity set (with respect to a potential theory associated with the Laplacian in $\mathbb{R}^{d}$ ). See for instance [9] and appendix $A$ in [27] for a discussion of the concept of Newtonian capacity and its relations with Wiener measure.

Assumption 3. There exist a positive trace-class selfadjoint operator $Q: \mathbb{R}^{d} \times \mathcal{H}_{t} \rightarrow \mathbb{R}^{d} \times \mathcal{H}_{t}$, such that, by denoting its maximum eigenvalue with $\lambda_{\max }$, one has

$$
\lambda_{\max }<1,
$$

and such that for any $(x, \gamma) \in \mathbb{R}^{d} \times \mathcal{H}_{t}$ the following inequality holds

$$
\begin{aligned}
& \mid \bar{\phi}\left(e^{i \pi / 4} x\right) \psi_{0}\left(e^{i \pi / 4} \gamma(t)\right.\left.+e^{i \pi / 4} x\right) e^{-i \int_{0}^{t} V\left(t-s, e^{i \pi / 4} \gamma(s)+e^{i \pi / 4} x\right) d s} \mid \\
& \leq e^{\frac{1}{2}((x, \gamma), Q(x, \gamma))-\frac{1}{2}|x|^{2}}, \quad(x, \gamma) \in \mathbb{R}^{d} \times \mathcal{H}_{t},
\end{aligned}
$$

Under the assumptions above, for any $\xi \in S_{d}$ the following integral

$$
\begin{gathered}
I_{t}^{\xi}(\phi, \psi):=e^{i d \pi / 4} \int_{\mathbb{R}^{d}} \int_{C_{t}} \bar{\phi}\left(e^{i \pi / 4} x\right) e^{-i \int_{0}^{t} V\left(t-s, e^{i \pi / 4} \gamma(s)+e^{i \pi / 4} x\right) d s} \\
\psi\left(e^{i \pi / 4} \gamma(t)+e^{i \pi / 4} x\right) e^{-e^{i 3 \pi / 4} \int_{0}^{t} \dot{\xi}(t-s)(\gamma(s)+x) d s} e^{-e^{i 3 \pi / 4} \xi(0)(\gamma(t)+x)+e^{i 3 \pi / 4} \xi(t) x} d W(\gamma) d x
\end{gathered}
$$

is well defined. Indeed it is an U-functional, as stated by the following theorem. 
Theorem 2. Let $\phi, \psi, V$ satisfying the assumptions 1, 2 and 3. Then the functional

$$
\xi \in S_{d} \mapsto F(\xi):=e^{-\frac{1}{2} \int_{-\infty}^{0} \xi(\tau)^{2} d \tau-\frac{1}{2} \int_{t}^{\infty} \xi(\tau)^{2} d \tau} I_{t}^{\xi}(\phi, \psi)
$$

is the T-transform of an Hida distribution.

Proof: We have to verify that $F$ is ray analytic, that is for all $\xi, \eta \in S_{d}$, the mapping

$$
z \in \mathbb{R} \mapsto F(\xi+z \eta)
$$

has an analytic continuation to an entire function on $\mathbb{C}$. In fact this follows from the analyticity of the integrand in (7), and from Fubini and from Morera theorems, which can be applied because of the assumption 3 .

The bounded growth of $F$ can be directly verified. Indeed, by assumption 3 , for $z \in \mathbb{C}$ and $\xi \in S_{d}$, one has:

$$
\begin{array}{r}
|F(z \xi)| \leq(2 \pi)^{d / 2} e^{\frac{|z|^{2}}{2} \int_{-\infty}^{0} \xi(\tau)^{2} d \tau+\frac{|z|^{2}}{2} \int_{t}^{+\infty} \xi(\tau)^{2} d \tau} \int_{\mathbb{R}^{d} \times C_{t}} e^{\frac{1}{2}((x, \gamma), Q(x, \gamma))} \\
e^{-\cos (3 \pi / 4+\theta)|z| \int_{0}^{t} \xi(s) d \gamma(s)} W(d \gamma) \frac{e^{-\frac{1}{2}|x|^{2}}}{(2 \pi)^{d / 2}} d x
\end{array}
$$

where $z=|z| e^{i \theta}$ and we have used the fact that

$$
\int_{0}^{t} \dot{\xi}(t-s)(\gamma(s)+x) d s+\xi(0)(\gamma(t)+x)-\xi(t) x=\int_{0}^{t} \xi(t-s) d \gamma(s),
$$

$\int_{0}^{t} \dot{\xi}(t-s) d \gamma(s)$ denoting Ito integration. By a direct computation we obtain the following bound

$$
\begin{array}{r}
|F(z \xi)| \leq(2 \pi)^{d / 2} \operatorname{det}(I-Q)^{-1 / 2} e^{\frac{|z|^{2}}{2} \int_{-\infty}^{0} \xi(\tau)^{2} d \tau+\frac{|z|^{2}}{2} \int_{t}^{+\infty} \xi(\tau)^{2} d \tau} \\
e^{\frac{1}{2} \cos ^{2}(3 \pi / 4+\theta)|z|^{2}\left(\left(0, v_{\xi}\right),(I-Q)^{-1}\left(0, v_{\xi}\right)\right),},
\end{array}
$$

where $\operatorname{det}(I-Q)$ is the Fredholm determinant of the operator $(I-Q)$ (which is well defined since $Q$ is a trace class operator), $(I-Q)^{-1}$ is its inverse, $v_{\xi}$ is the vector of $\mathcal{H}_{t}$ such that $\dot{v}_{\xi}(\tau)=\xi(t-\tau), \tau \in[0, t]$. By equation (5) the norm of the operator $(I-Q)^{-1}$ is finite, in particular $\left\|(I-Q)^{-1}\right\|=\left(1-\lambda_{\max }\right)^{-1}<\infty$ and we get

$$
\begin{aligned}
|F(z \xi)| \leq(2 \pi)^{d / 2} \operatorname{det}(I-Q)^{-1 / 2} e^{\frac{|z|^{2}}{2} \int_{-\infty}^{0} \xi(\tau)^{2} d \tau+\frac{|z|^{2}}{2} \int_{t}^{+\infty} \xi(\tau)^{2} d \tau} \\
e^{\frac{1}{2} \cos ^{2}(3 \pi / 4+\theta)|z|^{2}\left(1-\lambda_{\max }\right)^{-1} \int_{0}^{t} \xi(\tau)^{2} d \tau} \leq \alpha e^{\beta|z|^{2}\|\xi\|_{L_{d}^{2}}}
\end{aligned}
$$

where $\alpha=(2 \pi)^{d / 2} \operatorname{det}(I-Q)^{-1 / 2}$ and $\beta=\frac{1}{2} \max \left\{1,\left(1-\lambda_{\max }\right)^{-1}\right\}$. 
The functional $F(\xi)$, for suitable $\xi \in S_{d}$ can be interpreted as the functional integral representation of the solution of the Schrödinger equation in a weak sense.

Let us consider the Schrödinger equation with time dependent potential

$$
V_{\dot{\xi}}(\tau, x):=V(\tau, x)+\dot{\xi}(\tau) x,
$$

where $\dot{\xi} \in S_{d}$ is an external uniform force field dependent on time such that $\xi(\tau)=0$ for $\tau \neq(0, t)$. Let us denote with $H(\tau)$ the time dependent quantum Hamiltonian operator, given by

$$
H(\tau)=H_{0}+V_{\dot{\xi}}(\tau),
$$

$H_{0}$ being the free Hamiltonian $H_{0}=-\frac{1}{2} \Delta$, with its natural domain of self adjointness in $L^{2}\left(\mathbb{R}^{d}\right)$ [29]. The description of the domain of $H(\tau)$ is not trivial and it could depend on the time variable $t$.

The quantum dynamics associated to $H(t)$ is given by a two parameter family $U\left(t_{1}, t_{0}\right)$ of unitary operators on $L^{2}\left(\mathbb{R}^{d}\right), t_{0}, t_{1} \in \mathbb{R}$, such that

- $U\left(t_{2}, t_{1}\right) U\left(t_{1}, t_{0}\right)=U\left(t_{2}, t_{0}\right)$,

- $U\left(t_{0}, t_{0}\right)=I$,

- $U\left(t_{1}, t_{0}\right)$ is jointly strongly continuous in $t_{1}$ and $t_{0}$.

Under rather general assumptions on the potential (10) (see [30] theorem X.71), it is possible to prove that given any $\psi \in L^{2}\left(\mathbb{R}^{d}\right)$, the vector $\psi(t)=U(t, 0) \psi$ is a weak solution of the Schrödinger equation in the sense that for any $\phi \in L^{2}\left(\mathbb{R}^{d}\right)$ such that $\phi$ belongs to the domain of $H(t)$, the inner product $\langle\phi, \psi(t)\rangle$ is differentiable and

$$
i \frac{d}{d t}\langle\phi, \psi(t)\rangle=\langle H(t) \phi, \psi(t)\rangle
$$

The functional integral $I_{t}^{\xi}(\phi, \psi)$ given by equation $(7)$ is a candidate for the inner product $\langle\phi, \psi(t)\rangle$, as it satisfies an equation analogous to (12). More precisely the following holds:

Theorem 3. Let $\phi \in L^{2}\left(\mathbb{R}^{d}\right)$ belong to the domain of $H(\tau) \forall \tau \in[0, t]$. Let $\phi, \psi, V$ and $H(\tau) \phi, \psi, V$ satisfy the assumptions 1, 2 and $3 \forall \tau \in$ $[0, t]$. Then the functional integral $I_{t}^{\xi}(\phi, \psi)$ given by equation $(7)$ is a solution of the Schrödinger equation in the following weak sense:

$$
\begin{aligned}
& I_{0}^{\xi}(\phi, \psi)=\langle\phi, \psi\rangle \\
& i \frac{d}{d t} I_{t}^{\xi}(\phi, \psi)=I_{t}^{\xi}(H \phi, \psi)
\end{aligned}
$$


Proof: The first equality follows by the analyticity properties of the functions $\phi, \psi \in L^{2}\left(\mathbb{R}^{d}\right)$ (Assumption 1). Indeed for $z \in \mathbb{R}^{+}$, one has

$$
\int_{\mathbb{R}^{d}} \bar{\phi}(x) \psi(x) d x=z^{d} \int_{\mathbb{R}^{d}} \bar{\phi}(z x) \psi(z x) d x
$$

By the assumptions on $\phi, \psi$, as well as by Fubini and Morera theorems, the latter defines an analytic function of the variable $z \in D_{0, \pi / 4}$, which is continuous on $\bar{D}_{0, \pi / 4}$ and constant on $\mathbb{R}^{+}$, it follows then that it is constant over the whole sector $\bar{D}_{0, \pi / 4}$ and one gets

$$
\int_{\mathbb{R}^{d}} \bar{\phi}(x) \psi(x) d x=e^{i d \pi / 4} \int_{\mathbb{R}^{d}} \bar{\phi}\left(e^{i \pi / 4} x\right) \psi\left(e^{i \pi / 4} x\right) d x=I_{0}^{\xi}(\phi, \psi)
$$

For the proof of the second equality it is convenient to replace the expression $(7)$ of $I_{t}^{\xi}(\phi, \psi)$ with the following equivalent:

$$
\begin{array}{r}
I_{t}^{\xi}(\phi, \psi)=e^{i d \pi / 4} \int_{\mathbb{R}^{d}} \mathbb{E}\left[\bar{\phi}\left(e^{i \pi / 4} B(t)+e^{i \pi / 4} x\right) e^{-i \int_{0}^{t} V\left(s, e^{i \pi / 4} B(s)+e^{i \pi / 4} x\right) d s}\right. \\
\left.\psi\left(e^{i \pi / 4} x\right)\right] d x, \quad(14)
\end{array}
$$

where $\mathbb{E}$ denotes the expectation with respect to the Wiener measure. By applying Ito formula to the integrand

$$
f(t, B)=\bar{\phi}\left(e^{i \pi / 4} B(t)+e^{i \pi / 4} x\right) e^{-i \int_{0}^{t} V\left(s, e^{i \pi / 4} B(s)+e^{i \pi / 4} x\right) d s} \psi\left(e^{i \pi / 4} x\right),
$$

by integrating with respect to $d x$ on $\mathbb{R}^{d}$ and to the Wiener measure and finally by applying the Fubini theorem, one obtains:

$$
I_{t}^{\xi}(\phi, \psi)-I_{0}^{\xi}(\phi, \psi)=-i \int_{0}^{t} I_{\tau}^{\xi}(H(\tau) \phi, \psi) d \tau .
$$

\section{The SChröDinger Equation With POlynomial Potentials}

A particular example of states $\phi, \psi$ and potentials $V$ satisfying assumptions 1, 2 and 3 is described in [6]. Indeed let us consider two sets of vectors in $\mathcal{S}_{1}, \mathcal{S}_{2} \subset L^{2}\left(\mathbb{R}^{d}\right)$, where $\mathcal{S}_{1}$ is the subset of $S\left(\mathbb{R}^{d}\right)$ made of the functions $\psi: \mathbb{R}^{d} \rightarrow \mathbb{C}$ of the form

$$
\psi(x)=P(x) e^{-\frac{x^{2}}{2}(1-i)}, \quad x \in \mathbb{R}^{d},
$$

and $\mathcal{S}_{2}$ is the subset of $S\left(\mathbb{R}^{d}\right)$ made of the functions $\phi: \mathbb{R}^{d} \rightarrow \mathbb{C}$ of the form

$$
\phi(x)=Q(x) e^{-\frac{x^{2}}{2}(1+i)}
$$

where $P$ and $Q$ are polynomials with complex coefficients. As the Hermite functions form a complete orthonormal system in $L^{2}\left(\mathbb{R}^{d}\right)$, it 
is simple to verify that both $\mathcal{S}_{1}$ and $\mathcal{S}_{2}$ are dense in $L^{2}\left(\mathbb{R}^{d}\right)$. Moreover if $\phi \in S_{2}$, than $\bar{\phi} \in S_{1}$.

Let us consider a polynomial potential $V$ of the following form:

$$
V(\tau, x)=\frac{1}{2} x \Omega^{2} x+\alpha(\tau) V_{4 N}(x), \quad x \in \mathbb{R}^{d}
$$

where $\Omega^{2}$ is a symmetric positive $d \times d$ matrix, $\alpha:[0, t] \rightarrow \mathbb{R}^{d}$ is a continuous $^{2}$ function of the time variable and $V_{4 N}: \mathbb{R}^{d} \rightarrow \mathbb{R}$ is a homogeneous polynomial of degree $4 N, n \in \mathbb{N}$. Let $\Omega_{j}, j=1, \ldots d$, denote the eigenvalues of the matrix $\Omega$. Let us assume that the following inequalities are satisfied:

$$
\Omega_{j} t<\frac{\pi}{2}, \quad 1-\Omega_{j} \tan \left(\Omega_{j} t\right)>0
$$

Then it is possible to prove (see $[4,6]$ ), that the states $\phi \in \mathcal{S}_{2}, \psi \in \mathcal{S}_{1}$ and the time dependent potential $V$ satisfy the conditions 1, 2 and 3 . In particular the inequalities (18) implies condition 3. By theorem 2 the functional (8) is well defined and is the T-transform of a unique Hida distribution. Moreover, by theorem 3, given a vector $\xi \in S_{d}$, the functional

$$
\begin{gathered}
I_{t}^{\xi}(\phi, \psi):=e^{i d \pi / 4} \int_{\mathbb{R}^{d}} \int_{C_{t}} \bar{\phi}\left(e^{i \pi / 4} x\right) e^{(-1)^{N+1} i \int_{0}^{t} V_{4 N}(t-s, \gamma(s)+x) d s} \\
e^{\frac{1}{2} \int_{0}^{t}(\gamma(s)+x) \Omega^{2}(\gamma(s)+x) d s} e^{-e^{i 3 \pi / 4} \int_{0}^{t} \dot{\xi}(t-s)(\gamma(s)+x) d s} \psi\left(e^{i \pi / 4} \gamma(t)+e^{i \pi / 4} x\right) d W(\gamma) d x
\end{gathered}
$$

is a weak solution of the Schrödinger equation with potential $V_{\dot{\xi}}(\tau, x)=$ $V(\tau, x)+\dot{\xi}(\tau) x, \tau \in[0, t], x \in \mathbb{R}^{d}$, in the sense that it satisfies equation $(13)$.

Remark 2. An analogous result can be obtained by replacing the polynomial $V_{4 N}$ by another homogeneous potential of order $4 N$, continuous on the complement of a null capacity set, such as $V(\tau, x)=\frac{\alpha(\tau)}{|x|^{4 N}}$. Non regular potentials with this kind of singularities has also been handled by Nelson in [27] by means of a sequential approach and an analytic continuation technique.

\section{ACKNOWLEDGMENTS}

The hospitality of the Mathematics Institutes of Trento and of Bonn Universities is gratefully acknowledged, as well as the financial support of the Alexander von Humboldt Stiftung.

\footnotetext{
${ }^{2}$ this condition may be weakened
} 


\section{REFERENCES}

[1] S. Albeverio and Z. Brzeźniak. Finite-dimensional approximation approach to oscillatory integrals and stationary phase in infinite dimensions. J. Funct. Anal., 113 n.1, 177-244, 1993.

[2] S Albeverio, Z. Brzeźniak, Z. Haba, On the Schrödinger equation with potentials which are Laplace transforms of measures. Potential Anal. 9 (1998), no. $1,65-82$.

[3] S. Albeverio, R. Høegh-Krohn, S. Mazzucchi, Mathematical theory of Feynman path integrals. An Introduction. 2nd and enlarged edition. Lecture Notes in Mathematics, Vol. 523. Springer-Verlag, Berlin-New York (2008).

[4] S. Albeverio, S. Mazzucchi, Feynman path integrals for polynomially growing potentials, J. Funct. Anal. 221 no.1, 83-121 (2005).

[5] S. Albeverio, S. Mazzucchi, The time dependent quartic oscillator - a Feynman path integral approach. J. Funct. Anal. 238, no. 2, 471-488 (2006).

[6] S. Albeverio, S. Mazzucchi, An asymptotic functional-integral solution for the Schrödinger equation with polynomial potential. J. Funct. Anal. 257 (2009), no. 4, 1030-1052.

[7] R.H. Cameron, A family of integrals serving to connect the Wiener and Feynman integrals, J. Math. and Phys. 39, 126-140 (1960).

[8] M. De Faria, J. Potthoff, L. Streit, The Feynman integrand as a Hida distribution. J. Math. Phys. 32, 2123-2127 (1991).

[9] J.L. Doob, Classical potential theory and its probabilistic counterpart. Reprint of the 1984 edition. Classics in Mathematics. Springer-Verlag, Berlin, (2001).

[10] H. Doss, Sur une Résolution Stochastique de l'Equation de Schrödinger à Coefficients Analytiques. Commun. Math. Phys., 73, 247-264 (1980).

[11] D. Elworthy and A. Truman. Feynman maps, Cameron-Martin formulae and anharmonic oscillators. Ann. Inst. H. Poincaré Phys. Théor., 41(2), 115-142, 1984.

[12] R.P. Feynman, A.R. Hibbs. Quantum mechanics and path integrals. Macgraw Hill, New York, 1965.

[13] D. Fujiwara, N. Kumano-Go, The second term of the semi-classical asymptotic expansion for Feynman path integrals with integrand of polynomial growth. J. Math. Soc. Japan 58 (2006), no. 3, 837-867.

[14] D. Fujiwara, N. Kumano-Go, Feynman path integrals and semiclassical approximation. RIMS Kokyuroku Bessatsu, B5, Res. Inst. Math. Sci. (RIMS), Kyoto, 2008, 241-263.

[15] Gross L., Abstract Wiener Spaces, Proc. $5^{\text {th }}$ Berkeley Symp. Math. Stat. Prob. $2,(1965) 31-42$.

[16] M. Grothaus, D.C. Khandekar, J.L. da Silva, L. Streit, The Feynman integral for time-dependent anharmonic oscillators. J. Math. Phys. 38, no. 6, 3278-3299 (1997).

[17] M. Grothaus, L. Streit, A. Vogel, Feynman integrals as Hida distributions: the case of non-perturbative potentials. to appear in Astérisque, special issue in honor of Jean-Michel Bismut's sixtieth birthday, 2010.

[18] T. Hida, White noise approach to Feynman integrals. J. Korean Math. Soc. 38, no. 2, 275-281 (2001).

[19] T. Hida, H.H. Kuo, J. Potthoff, L. Streit, White Noise. An infinite dimensiona calculus. Kluwer, Dordrecht (1995). 
[20] G.W. Johnson and M.L. Lapidus, The Feynman integral and Feynman's operational calculus. Oxford University Press, New York (2000).

[21] G. Kallianpur, D. Kannan, R.L. Karandikar Analytic and sequential Feynman integrals on abstract Wiener and Hilbert spaces, and a Cameron Martin Formula. Ann. Inst. H. Poincaré, Prob. Th. 21 (1985), 323-361.

[22] T. Kuna, L. Streit, W. Westerkamp, Feynman integrals for a class of exponentially growing potentials, J. Math. Phys. 39, no. 9, 4476-4491 (1998).

[23] Kuo H.H., Gaussian Measures in Banach Spaces, Lecture Notes in Math., Springer-Verlag Berlin-Heidelberg-New York, (1975).

[24] A. Lascheck, P. Leukert, L. Streit, W. Westerkamp, Quantum Mechanical Propagators in Terms of Hida Distribution, Rep. Math. Phys. 33, 221-232 (1993).

[25] S. Mazzucchi, Feynman path integrals for the inverse quartic oscillator, J. Math. Phys. 49, 9 (2008), 093502 (15 pages)

[26] S. Mazzucchi, Mathematical Feynman Path Integrals and Applications. World Scientific Publishing, Singapore (2009)

[27] E. Nelson, Feynman integrals and the Schrödinger equation. J. Math. Phys. 5 (1964), 332-343.

[28] J. Potthoff, L. Streit, A characterization of Hida distributions. J. Funct. Anal. 101, no. 1, 212-229 (1991).

[29] M. Reed, B. Simon, Methods of modern mathematical physics. I. Functional analysis. Second edition. Academic Press, Inc. [Harcourt Brace Jovanovich, Publishers], New York, 1980.

[30] M. Reed, B. Simon, Methods of modern mathematical physics. II. Fourier analysis, self-adjointness. Academic Press [Harcourt Brace Jovanovich, Publishers], New York-London, 1975.

[31] B. Simon, Coupling Constant Analyticity for the Anharmonic Oscillator. Annals of Physics 58, 76-136 (1970).

[32] K. Yajima, Smoothness and Non-Smoothness of the Fundamental Solution of Time Dependent Schrödiger Equations, Commun. Math. Phys. 181, 605-629 (1996).

Dip. Matematica, Università di Trento, 38050 Povo (I) 